\title{
Kernos
}

Revue internationale et pluridisciplinaire de religion grecque antique

1 | 1988

Varia

\section{Archéologie et religion : problèmes et méthode}

\section{Robert Laffineur}

Edition électronique
URL : http://journals.openedition.org/kernos/103

DOI : $10.4000 /$ kernos. 103

ISSN : 2034-7871

\section{Éditeur}

Centre international d'étude de la religion grecque antique

Édition imprimée

Date de publication : 1 janvier 1988

ISSN : 0776-3824

\section{Référence électronique}

Robert Laffineur, "Archéologie et religion : problèmes et méthode », Kernos [En ligne], 1 | 1988, mis en ligne le 31 janvier 2011, consulté le 22 avril 2019. URL : http://journals.openedition.org/kernos/103 ; DOI : 10.4000/kernos.103 
Kernos, 1(1988), p. 129-140.

\section{ARCHÉOLOGIE ET RELIGION : PROBLÈMES ET MÉTHODE}

On a coutume de confronter, au plan de l'épistémologie, la valeur respective des sources matérielles et des sources textuelles dans la connaissance des civilisations passées et de leurs divers aspects, notamment de la religion. Si ces témoignages sont à l'évidence complémentaires, on a parfois tendance à reconnaître aux seconds une sorte de supériorité qui tiendrait à leur caractère plus explicite. Mais on peut inversement accorder une importance plus grande aux vestiges matériels en raison de leur universalité, non seulement dans le temps - les longues périodes préhistoriques sont privées par définition de témoignages écrits - et dans l'espace - les nombreuses civilisations sans écriture -, mais aussi dans les multiples aspects qu'ils révèlent d'une société, et en raison de leur nature même, qui en fait des sources primaires et objectives. La question a été admirablement traitée il y a quelques années par Philippe Bruneau ${ }^{1}$ et mon intention n'est pas ici d'ouvrir une nouvelle fois ce débat dans un esprit partisan, mais de tenter d'éclairer davantage la valeur des témoignages archéologiques au départ de considérations empruntées au domaine religieux, après avoir apporté quelques précisions utiles sur la complémentarité et sur la spécificité des sources.

Et d'abord une brève réflexion concernant les périodes historiques. Le fonctionnement et l'histoire d'un sanctuaire comme celui d'Apollon à Delphes sont, malgré de nombreuses lacunes, assez bien connus dans l'ensemble, de même que sa topographie, grâce principalement au témoignage des auteurs anciens et des inscriptions. Les vestiges mis au jour n'apportent guère, outre précisément les inscriptions dont certains sont couverts et qui en sont les supports indispensables, de données complémentaires, sauf bien entendu pour la connaissance de l'architecture et de la décora-

1 Ph. BRUNEAU, Sources textuelles et vestiges matériels : réflexions sur l'interprétation archéologique, in Mélanges helléniques offerts à Georges Daux, Paris, 1974, p. 33-42. 
tion des monuments eux-mêmes. La chronologie ne peut pas toujours être précisée par les données archéologiques, comme le montre spécialement le cas du Trésor des Athéniens. Le seul apport des vestiges - mais il est loin d'être négligeable - concerne les monuments que les témoins, pas toujours contemporains, ont mal situés, qu'ils n'ont pas vus ou qu'ils ont simplement omis de mentionner ou de décrire. On mesure ici les limites du témoignage secondaire et la chose est bien mise en évidence, à Delphes encore, par la confrontation des vestiges conservés et du témoignage de Pausanias pour le premier secteur de la voie sacrée, comme l'ont montré Jean Pouilloux et Georges Roux qui amènent à s'interroger sur la fiabilité du témoignage écrit ${ }^{2}$, ou par exemple par Claude Vatin qui a proposé récemment d'identifier un nouveau monument, le portique des Tégéates, dont on possède les restes bien identifiables, mais à propos duquel les contemporains sont restés muets ${ }^{3}$. Les témoignages littéraires peuvent révéler des limites comparables concernant les détails de certains monuments. La décoration sculptée des frontons du temple du IVe siècle en donne une illustration particulièrement suggestive : la mention du Soleil couchant dans la description de Pausanias, la $\delta \dot{\sigma} \sigma ı \varsigma_{\tau} \varepsilon{ }^{\prime} H \lambda$ íov$^{4}$, n'est peut-être pas à comprendre, selon Francis Croissant, comme faisant partie de l'énumération des figures visibles dans les tympans, au même titre que les représentations d'Artémis, Léto, Apollon et les Muses et de Dionysos et les Thyiades, puisqu'aucun fragment attribuable à un char d'Hélios ne se trouve dans les vestiges conservés, mais plutôt comme une indication d'orientation, qui introduit la seconde partie

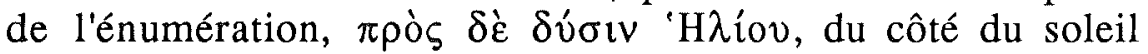
couchant, comme si le Périégète avait «confondu, au moment de la rédaction, une simple notation topographique avec le contenu iconographique $^{5}$ ».

Les lacunes peuvent concerner inversement les sources matérielles et le site de Delphes offre à nouveau un exemple typique. Il s'agit du célèbre adyton, situé au fond de la cella du grand temple

2 J. POUILlOUX et G. ROUX, Enigmes à Delphes, Paris, 1963, p. 3-68.

3 Cl. VATIN, Monuments votifs de Delphes IV, Le portique de Tégée, dans $B C H$, 105(1981), p. 453-459.

4 PAUSANIAS, $\mathrm{X}, 19,4$.

5 Fr. CROISSANT, Les frontons du temple du IVe siècle à Delphes : premier: essais de restitution, in $R A$ (1980), p. 11. 
d'Apollon. Les restes qui en sont conservés in situ sont pratiquement inexistants, puisqu'ils se réduisent à un vide, et nous ne disposons que de témoignages littéraires, la plupart indirects, pour reconstituer l'aspect de cette partie de l'édifice qui jouait pourtant un rôle essentiel dans le fonctionnement de l'oracle. Ces sources suggèrent, comme l'indique Georges Roux, «une simple fosse quadrangulaire peu profonde ou plus exactement une échancrure dans le pavage du temple laissant apparaître en contre-bas le sol du Parnasse» et la disparition totale des vestiges d'une telle installation ne peut surprendre, puisque selon les termes mêmes du savant français «rien ne laisse moins de ruines qu'un trou $!^{6} \gg$. L'inventaire du mobilier de l'adyton nous est connu, mais la manière dont les divers éléments étaient disposés reste hypothétique et l'existence de la fameuse ouverture dans le sol, le $\chi \alpha \dot{\sigma} \sigma \mu \alpha \gamma \hat{\eta} \varsigma$, d'où s'échappait la prétendue vapeur inspiratrice, ne peut être confirmée archéologiquement ${ }^{7}$.

Voilà un ensemble de relations complexes qui dépasse largement l'impression habituelle de la complémentarité élémentaire des deux catégories de sources.

Si l'on s'intéresse d'autre part à la plus haute antiquité du sanctuaire, on doit bien convenir en revanche que notre connaissance est excessivement fragmentaire. La disproportion quantitative et qualitative des connaissances entre la période préhistorique ou protohistorique et les siècles ultérieurs apparaît toujours à l'évidence, en matière de religion comme dans bien d'autres domaines. Les vestiges préhistoriques de nature cultuelle se limitent à Delphes à un fragment de rhyton zoomorphe en calcaire, trouvé dans la zone du grand temple ${ }^{8}$, et à des séries de figurines divines en terre cuite exhumées dans le sanctuaire du bas, réservé dans la suite à Athéna ${ }^{9}$. Des éléments aussi isolés ne permettent guère, même

6 G. ROUX, Delphes, son oracle et ses dieux, Paris, 1976, p. 110.

7 Sur la reconstitution de l'adyton, cf. ROUX, op. cit., p. 100-117.

8 P. PERDRIZET, Fouilles de Delphes, V, [1], Paris, 1908, p. 3-5 et fig. 13, et Th. HOMOLLE, Fouilles de Delphes, IV, 1, Art primitif. Art archaique du Péloponnèse et des îles, Paris, 1909, p. 3 et fig. 2.

9 R. DEMANGEL, Fouilles de Delphes, II, Le sanctuaire d'Athéna Pronaia, 3, Topographie du sanctuaire, Paris, 1926, p. 14-28 et fig. 16-32. Sur l'occupation du site au 2e millénaire, cf. ROUX, op. cit., p. 20-21. 
avec le secours des données de la légende qui se rapportent incontestablement aux phases anciennes de l'histoire du sanctuaire, de restituer autre chose qu'une occupation du site durant la seconde moitié du deuxième millénaire. Et on peut effectivement être tenté de conclure, même en tenant compte des réserves qui viennent d'être indiquées, qu'un phénomène de nature essentiellement idèologique ne peut être connu qu'imparfaitement à la lumière du seul éclairage matériel. La situation n'est heureusement pas souvent aussi défavorable et l'apport de l'archéologie, quand il ne peut bénéficier de l'appoint des témoignages écrits, est loin d'être insignifiant. C'est le cas de la religion de l'âge du Bronze, que l'absence de textes condamne à première vue à une approche tronquée et à laquelle je voudrais consacrer l'essentiel de la présente communication. La publication récente de l'excellent rapport définitif des fouilles anglaises du sanctuaire de Phylacopi de Mélos invite à une telle réflexion, autant par la matière traitée que par le titre choisi pour le volume par Colin Renfrew : The Archaeology of Cult.

Le savant anglais définit bien en préambule les deux attitudes qui prévalent quand il s'agit d'apprécier l'apport des données archéologiques à la connaissance des phénomènes religieux : un scepticisme et une prudence extrêmes, appliqués également à l'approche du contexte social, qui sont dictés par la conviction de l'incapacité des vestiges matériels à exprimer les croyances humaines et qui limitent en conséquence la validité des interprétations archéologiques, et un optimisme ou une intuition naïve qui amènent à considérer que les données matérielles observées peuvent valablement être l'objet d'une interprétation qui fasse appel au «libre exercice du don créatif de l'esprit humain pour l'imagination ${ }^{10} \gg . \mathrm{La}$ première attitude n'est qu'une manifestation d'une conception hiérarchisée de la valeur des inférences et déductions tirées du matériel archéologique, suivant qu'elles concernent, en ordre de validité décroissante, les phénomènes de techniques de production, ceux qui ont trait à l'économie et à la subsistance, les institutions socio-politiques et les institutions religieuses. La seconde attitude, souvent excessive, trouve habituellement sa justification dans un examen comparatif qui fait abstraction des critères de lieu et dans la

10 C. RENFREW et al., The Archaeology of Cult. The Sanctuary at Phylakopi, Londres, 1985 (BSA Suppl. XVIII), p. 1-4. 
conviction que, pour une même région, l'évolution des croyances religieuses et de leurs manifestations est caractérisée essentiellement par la continuité et que celle-ci autorise donc à associer au niveau de l'interprétation des témoignages d'époques différentes - les sources de l'âge du Bronze et les sources écrites de la Grèce historique en l'occurrence ${ }^{11}$.

Colin Renfrew met bien en évidence également les deux étapes successives de la démarche, l'identification des éléments cultuels et leur interprétation - c'est-à-dire principalement la détermination de leur fonction - et la nécessité de ne procéder à la seconde qu'après que la première a pu être établie avec des critères de certitude ou de probabilité suffisants ${ }^{12}$. Or c'est précisément dans cette première opération d'identification des manifestations matérielles du culte et des croyances que se situe, pour les périodes sans écriture, le seul fondement possible d'une connaissance objective. On mesure bien ici l'importance des témoins archéologiques et de leur examen approfondi, et la relation privilégiée et intime qui s'établit dans ces conditions entre religion et archéologie.

L'acquisition des connaissances n'a pas toujours été, loin s'en faut, obtenue suivant le processus de cheminement logique et inductif qui vient d'être défini et le constat en a été récemment dressé par Susan Guettel Cole dans une contribution intitulée Archaeology and Religion. A y regarder de près, on s'aperçoit que certaines des interprétations générales les plus couramment admises en matière de religion créto-mycénienne sont loin d'être confirmées par le témoignage des trouvailles archéologiques. C'est le cas de l'idée de l'existence d'une divinité primitive de la fertilité associée au monde animal et végétal, de l'introduction ultérieure par les Indo-européens d'un panthéon dominé par une divinité ouranienne et de la fusion de ces deux composantes aux époques historiques. Chacune de ces conceptions synthétiques résulte d'une intuition et d'une généralisation hâtive qu'une analyse systématique et complète des vestiges et de leur contexte précis amène à nuancer ${ }^{13}$. La première repose sur le

11 Contre l'idée de continuité, cf. S.G. COLE, Archaeology and Religion, in Contributions to Aegean Archaeology. Studies in Honor of William A. McDonald, Minneapolis, 1985, p. 54-55.

12 RENFREW, op, cit, p. 3.

13 COLE, op. cit., p. $49-59$ (spécialement p. 50-52). 
type majoritairement féminin des statuettes réputées divines mises au jour dans les fouilles, mais on observe en réalité l'existence de nombreuses figures masculines - sans compter les représentations d'individus de sexe indéterminé - et des indices non négligeables d'un symbolisme qui exprime des valeurs essentiellement masculines. Quant à Zeus, il est loin d'apparaître comme la divinité principale des Mycéniens et c'est plutôt à Poseidon que semble réservée la prééminence, si l'on en juge par les mentions des divinités dans les tablettes en linéaire $\mathrm{B}$.

L'identification des données matérielles de base, qui alimentent les appréciations et les conclusions plus générales, se fait trop rarement avec la rigueur nécessaire. Comment décider par exemple et dans quelles circonstances, pour reprendre un cas simple cité par Renfrew ${ }^{14}$, si telle figurine animale en terre cuite était une offrande à la divinité ou un simple jouet destiné aux enfants ? Comment établir, autrement que par une conviction largement intuitive fondée le plus souvent sur des témoignages éloignés, que les spécimens ou les images de double hache ou de cornes de consécration de Crète minoenne ont obligatoirement et dans n'importe quel contexte une valeur cultuelle ? Il y a là une sorte d'automatisme, dicté avant tout par l'érudition, qui s'impose à la réalité des faits observables, un cheminement à rebours de la pensée qui applique une interprétation issue de sources extérieures à des vestiges matériels qui ne sont pas nécessairement liés au culte ou qui n'y sont pas liés de la même manière ou dans les mêmes conditions. Un tel réflexe amènera par exemple, au plan des détails, à associer immédiatement les images de chouette à Athéna alors qu'elles peuvent être chargées de connotations différentes et variées en dehors du domaine attique et classique $^{15}$. Mais que dire alors d'une tentative d'interprétation religieuse plus générale d'un ensemble de vestiges comme celle qu'a proposée récemment Gösta Säflund à propos des trouvailles d'Akrotiri de Théra :

The large numbers of eating and drinking vessels stored in the buildings, and the conspicuous presence of vessels and utensils of a religious character in form and decoration, indicate that the buildings were intended

14 Op. cit., p. 2.

15 Sur le symbolisme de la chouette à l'époque mycénienne, cf. R. LAFFINEUR, in $A C, 50(1981)$, p. $432-444$. 
for the preparing and celebrating of sacrificial banquets of a non-private character - in other words : that the buildings had a cultic character and function...

ou

As regards the finding of mill installations and of loomweights in most of the buildings, we may assume, on the analogy of Greek religious customs, that it was the priestesses, preferably the young ones, who on the occasion of the festivals did the milling of the flour and the aromatics, as well as the weaving of the sacred robes ${ }^{16}$.

Cela ne signifie nullement qu'il faille renoncer à toute tentative d'interprétation, mais seulement que la seule démarche valide est celle qui s'intéresse aux données archéologiques pour en extraire tout le message dont elles sont porteuses. Les objets eux-mêmes apportent une partie de ce message, mais l'essentiel en est transmis par le contexte de trouvaille entendu au sens le plus large. Mais il s'agit bien du contexte propre à chaque cas et on ne peut, en l'absence de données d'association explicites pour tel document isolé, admettre et identifier sa fonction cultuelle sur base de la seule répétition de la trouvaille et en utilisant des contextes voisins plus explicites, même si ceux-ci offrent des critères de probabilité suffisants en ce qui les concerne ${ }^{17}$. Il faut donc que les conditions favorables soient réunies dans chaque circonstance spécifique, c'est-àdire que la mise au jour ait été effectuée avec la rigueur indispensable, notamment en matière d'enregistrement des données, et que le contexte de trouvaille ne soit pas équivoque ou lacunaire. C'est aujourd'hui une double exigence remplie sur de nombreux sites et qui permet, sans le secours des sources écrites, de disposer de constatations objectives dans les différents domaines de l'activité cultuelle.

16 G. SÄFLUND, Cretan and Theran Questions, in Sanctuaries and Cults in the Aegean Bronze Age. Proceedings of the First International Symposium at the Swedish Institute in Athens, 12-13 May 1980, Stockholm, 1981, p. 193 et 195.

17 Un exemple extrême de semblable généralisation chez SÄFLUND, op. cit., p. 205-206, n. 77 , à propos de la célèbre table d'offrande peinte d'images de dauphins trouvée en place sur l'appui d'une fenêtre d'une pièce de la Maison de I'Ouest, à Akrotiri, donnant sur une place publique : «Thus, the window may have served as a kind of window of appearances, and such may, as a matter of fact, have been the raison d'être of all the wide windows of the Akrotiri complex, as well as of those of the Cretan palatial complexes, as illustrated by the Knossian miniature frescoes and also by the Arkhanes house model». 
L'inventaire de ces données ne peut pas être dressé ici ${ }^{18}$, mais on sait bien que l'on dispose de vestiges souvent abondants concernant les sanctuaires publics et leur équipement mobilier ${ }^{19}$, le culte domestique ${ }^{20}$, les instruments du culte ${ }^{21}$, les actes du culte, sacrifices ou libations par exemple ${ }^{22}$, les offrandes votives ${ }^{23}$, c'est-à-dire à propos de la plupart des manifestations matérielles des croyances. A propos des actes du culte, on note que les vestiges apportent parfois un témoignage extrêmement précis sur des pratiques qui, pour exceptionnelles qu'elles ont dû être, car imposées par des circonstances particulières, n'en constituent pas moins des indices révélateurs de la force de conviction des croyances. C'est le cas des restes de sacrifice humain mis au jour dans le sanctuaire d'ArchanesAnemospilia ${ }^{24}$ et des vestiges de pratiques d'anthropophagie rituelle révélés à Cnossos, à propos desquelles $\mathrm{P}$. Warren a bien mis en

18 Pour la religion minoenne, on se reportera en priorité au récent recueil bibliographique de I.-M. RUUD, Minoan Religion. A Bibliography, Oslo, 1980 (avec index analytique). On rappellera aussi qu'une place est réservée à la religion des périodes préhistoriques et protohistoriques dans le programme Mentor, Bibliographie critique informatisée de la religion grecque, en cours de réalisation à l'Université de Liège.

19 A propos des sanctuaires publics, voir en dernier lieu COLE, op. cit., p. 5253, Sanctuaries and Cults in the Aegean Bronze Age. Proceedings of the First International Symposium at the Swedish Institute in Athens, 12-13 May, 1980, Stockholm, 1981, passim et B. RUTKOWSKI, Cult Places of the Aegean, Londres et New Haven, 1986. Pour les statues de culte, on verra la récente publication des trouvailles du sanctuaire d'Aghia Irini de Keos: M.E. CASKEY, Keos, II, The Tempel at Ayia Irini, I, The Statues, Princeton, 1986.

20 G.C. GESELL, Town, Palace and House Cult in Minoan Crete, Göteborg, 1985.

21 Cf. RUUD, op. cit., p. 62 (§13).

22 RUUD, op. cit., p. 59-60 (\$9). A propos des sacrifices, des libations et des autres pratiques cultuelles, voir tout récemment N. MARINATOS, Minoan Sacrificial Ritual. Cult Practice and Symbolism, Stockholm, 1986.

23 Les dépôts votifs sont très nombreux, spécialement dans les grottes et les sanctuaires de sommet. Cf. à ce propos en dernier lieu St. HILLER, Das minoische Kreta nach den Ausgrabungen des letzten Jahrzehnts, Vienne, 1977, passim.

24 Le rapport le plus synthétique à propos de cette trouvaille a été publié par I. SALELLARAKIS et E. SAPOUNA-SAKELLARAKI dans le National Geographic Magazine, février 1981, p. 204-222. A propos des sacrifices humains, cf. aussi E. PROTONOTARIOU-DEILAKI, $\theta o \lambda \omega \tau \grave{\alpha} \varsigma \tau \dot{\alpha} \varphi \sigma \zeta K \alpha \zeta \dot{\alpha} \rho \mu \alpha \zeta$, in ArchAnAth, 2(1969), p. 3-5 et L. BAUMBACH, An Examination of the Evidence for a State of Emergency at Pylos c. 1200 BC from the Linear B Tablets, in Res Mycenaeae. Akten des VII. Internationalen Mykenologischen Colloquiums in Nürnberg, Göttingen, 1983, p. 34. 
évidence la pertinence d'une interprétation fondée sur des témoignages littéraires postérieurs ${ }^{25}$.

On rappellera aussi que ces mêmes aspects matériels sont accessibles également par les images contemporaines qui nous en sont parvenues. C'est le vaste domaine de l'iconographie qui est concerné ici et on connaît les difficultés particulières que posent l'interprétation des scènes cultuelles, mais surtout leur identification en tant que scènes de culte, et la tentation fréquente de dépasser les limites d'une analyse objective. L'iconographie donne par ailleurs un accès direct à des aspects de la vie religieuse dont les vestiges matériels ne peuvent enregistrer le souvenir, comme les gestes accomplis au cours des cérémonies, le déroulement des cérémonies elles-mêmes, le personnel du culte et, dans une certaine limite, le panthéon ${ }^{26}$. La forme figurée que prennent certains objets exhumés dans les fouilles peut même trahir, dans quelques rares cas, certaines croyances que l'on ne s'attend à connaître que par les témoignages écrits.

C'est le cas de la croyance en une forme de survie après la mort. Elle peut, semble-t-il, être déduite de l'association au mobilier funéraire des tombes du début de l'époque mycénienne d'images d'animaux dont le mode de vie se caractérise par des habitudes d'hibernation (abeille, triton, cervidé), par une faculté de régénération (poulpe) ou par un développement en une succession de métamorphoses, des naissances successives en quelque sorte (papillon, chrysalide ou cigale, grenouille), de même que par celle de figures d'animaux auxquels on reconnaît volontiers une valeur protectrice (chouette, aigle). C'est l'observation que j'ai cru pouvoir faire récemment, après avoir noté que ces représentations, loin d'être isolées, constituent des séries, et que la répétition fréquente du même motif et le matériau généralement précieux et inaltérable dans lequel sont modelées les fígures devaient sans doute contribuer à renforcer l'efficacité et la durée de ce symbolisme de l'accession à

25 P. WARREN, Minoan Crete and Ecstatic Religion, Preliminary Observations on the 1979 Excavations at Knossos, in Sanctuaries and Cults..., p. 155-167.

26 RUUD, op. cit., p. 56-59 (§ 3-6). Pour une méthodologie de l'approche iconographique, cf. en dernier lieu N. MARINATOS, Art and Religion in Thera. Reconstructing a Bronze Age Society, Athènes, 1984, p. 7-10. 
une vie nouvelle après la mort ${ }^{27}$. Si l'observation de ces particularités n'est attestée avec certitude qu'aux époques historiques, dans les textes des auteurs anciens, il n'en reste pas moins qu'il s'agit là de caractéristiques aisément reconnaissables, sans le recours à l'expérimentation scientifique, dans le cadre des activités d'une vie quotidienne étroitement dépendante de la nature. Cette même croyance en une forme de survie paraît trouver une autre expression dans l'utilisation essentiellement funéraire des rhytons zoomorphes dans le domaine mycénien. La majorité des contextes de trouvaille indiquent que l'objet, souvent réduit à la tête, avait probablement une fonction dans les croyances funéraires. Ne s'agissait-il pas d'un sacrifice symbolique de substitution dont l'image était rendue perpétuelle par la figuration de sa victime - on observe ici aussi, bien que plus rarement, l'utilisation du métal précieux pour le modelage

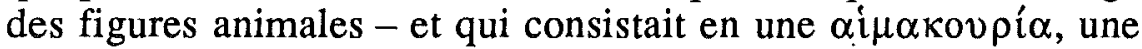
libation de sang, liquide de vie par excellence, ou d'un substitut quelconque destiné à communiquer symboliquement ses vertus régénératrices au sol et au défunt qui s'y trouve inhumé28 ? N'y a-til pas là l'équivalent du sacrifice pars pro toto, dont le crâne de bovidé découvert à l'entrée de la chambre latérale de la tholos $\mathrm{A}$ d'Archanes de date MR IIIA constitue la version réelle et ponctuelle ${ }^{29}$, sacrifice dont l'usage n'était sans doute pas aussi exceptionnel dans le cadre du rituel funéraire?

27 R. LAFFINEUR, Iconographie minoenne et iconographie mycénienne à l'époque des tombes à fosse, in L'iconographie minoenne. Actes de la Table Ronde d'Athènes (21-22 avril 1983), Paris, 1985 (BCH Suppl. XI), p. 245 266.

28 La décoration peinte du sarcophage d'Aghia Triada semble bien nous donner un exemple précis de cette pratique de régénération du sol : le sang du taureau sacrifié s'écoule dans un récipient qui est probablement un rhyton conique à moitié enfoncé dans le sol plutôt qu'un baquet tronconique (cf. Ch.R. LONG, The Ayia Triadha Sarcophagus. A Study of Late Minoan and Mycenaean Funerary Practices and Beliefs, Göteborg, 1974, p. 63 et n. 37).

29 R. LAFFINEUR, Fécondité et pratiques funéraires en Egée à l'âge du Bronze, in Archaeology and Fertility Cult in the Ancient Mediterranean. Papers presented at the First International Conference on Archaeology of the Ancient Mediterranean, Malta 2-5 September 1985, Amsterdam, 1986, p.79-96 et Weitere Beiträge zur Symbolik im mykenischen Bestattungsritual, in Kolloquium zur Ägäischen Vorgeschichte, Mannheim, 20.-22.2.1986, Mannheim, 1987 (Schriften des Deutschen Archäologen-Verbanck, IX), p. 125-132. Pour la trouvaille d'Archanes et les sacrifices d'animaux, voir I. SAKELLARAKIS, Das Kuppelgrab A von Archanes und das kretisch-mykenische Tieropferritual, in PrZ, 45(1970), p. 135-219. Pour les sacrifices, cf. aussi COLE, op. cit., p. $55-56$. 
Les vestiges archéologiques ne donnent pas seulement ainsi accès aux croyances qui se manifestent dans les pratiques du culte; ils permettent également de saisir, d'une manière plus générale, la place qu'occupe le culte dans la société. Cette hiérarchie à trois niveaux - matériel, idéologique et sociologique - a bien été mise en évidence par Colin Renfrew et les fouilles du sanctuaire de Phylacopi ont livré sur ce point des indices intéressants ${ }^{30}$. Mais c'est là un vaste domaine qui implique la confrontation de données multiples et qui se situe au-delà des limites que je m'étais fixées ici.

Les périodes préhistoriques se révèlent ainsi, malgré l'absence de témoignages écrits, riches de perspectives dans l'étude de la religion. Il n'y a pas là un paradoxe, mais simplement une conséquence de l'état lacunaire des sources dont nous disposons, qui impose une rigueur plus grande. Celle-ci doit s'appliquer à la mise au jour des structures et des objets, ainsi qu'à l'examen minutieux des contextes de trouvaille. Elle doit ensuite s'appliquer à l'interprétation, où la condition essentielle me paraît être de faire abstraction, en un premier temps, de tout l'acquis dû à l'érudition pour se concentrer exclusivement sur les données matérielles objectives directement observables. L'archéologie propose ici de nombreux modèles de méthodologie. Ne convient-il pas, quand on étudie un matériel céramique exhumé dans une fouille, de commencer par examiner ses particularités techniques et les données de la stratigraphie avant de tenter d'en donner un classement chronologique, plutôt que d'imposer à ce matériel, sur base d'un examen comparatif superficiel, des données acquises, sous prétexte qu'elles semblent justifiées par les résultats de recherches menées antérieurement sur d'autres sites ? Et la préhistoire ou la protohistoire donnent ici une sorte de leçon à cause précisément de l'unicité des sources et de la nécessité de leur exploitation plus systématique, de la même manière qu'elles imposent une plus grande rigueur en matière de méthodes de fouille par exemple. Les exigences d'une telle situation devraient être davantage prises en compte dans l'étuảe des phénomènes religieux des périodes historiques qui montre fréquemment une tendance à se reporter trop rapidement vers les autres catégories de sources, sans avoir cherché d'abord à épuiser le message des données archéologiques. Ce qui paraît une évidence et une obligation dans un domaine comme celui des productions céramiques, où

30 RENFREW, op. cit., p. 25-26 et 389-391. 
les témoignages écrits font défaut quelles que soient les périodes, devrait-il être négligé dans l'approche d'une composante de civilisation caractérisée par la multiplicité des sources?

On objectera que les pratiques cultuelles de l'âge du Bronze égéen ne constituaient pas un ensemble cohérent, une véritable religion organisée de manière systématique autour d'un système de croyances, au point que l'on devrait en désigner les principales manifestations par des termes plus appropriés à pareille situation, en parlant d'«activité rituelle» au lieu de culte et de «lieu d'activité rituelle» plutôt que de temple ${ }^{31}$, et que leur contenu idéologique ne présentait pas en conséquence une complexité équivalente à celui de la religion grecque des époques historiques. Il y a là sans doute une différence non négligeable - encore que la religion grecque soit dominée également par les particularismes locaux et marquée le plus souvent par l'absence d'unité -, mais elle ne change rien, d'une époque à l'autre, en ce qui concerne les manifestations matérielles du rituel et les traces qu'il a laissées dans l'environnement archéologique. C'est la raison de la similitude fondamentale de l'approche scientifique du phénomène et de la nécessité d'y procéder avec une rigueur égale, quels que soient les temps et les lieux.

Université de Liège

Robert LAFFINEUR

Place du XX-Août 32

B - 4000 LIEGE

31 COLE, op. cit., p. 49 et n. 1, p. 57. 